\title{
Microscopy images segmentation algorithm based on shearlet
} neural network

\author{
Nemir Ahmed Al-Azzawi \\ Department of Mechatronics Engineering, University of Baghdad, Iraq
}

\begin{tabular}{l} 
Article Info \\
\hline Article history: \\
Received Sep 13, 2020 \\
Revised Nov 21, 2020 \\
Accepted Dec 4, 2020 \\
\hline
\end{tabular}

\section{Keywords:}

Electron microscopy image

Image segmentation

Machine learning

Neural network

Shearlet transform

\begin{abstract}
Microscopic images are becoming important and need to be studied to know the details and how-to quantitatively evaluate decellularization. Most of the existing research focuses on deep learning-based techniques that lack simplification for decellularization. A new computational method for the segmentation microscopy images based on the shearlet neural network (SNN) has been introduced. The proposal is to link the concept of shearlets transform and neural networks into a single unit. The method contains a feedforward neural network and uses a single hidden layer. The activation functions are depending on the standard shearlet transform. The proposed SNN is a powerful technology for segmenting an electron microscopic image that is trained without relying on the pre-information of the data. The shearlet neural networks capture the features of full accuracy and contextual information, respectively. The expected value for specific inputs is estimated by learning the functional configuration of a network for the sequence of observed value. Experimental results on the segmentation of twodimensional microscopy images are promising and confirm the benefits of the proposed approach. Lastly, we investigate on a challenging datasets ISBI 2012 that our method (SNN) achieves superior outcomes when compared to classical and deep learning-based methods.
\end{abstract}

This is an open access article under the CC BY-SA license.

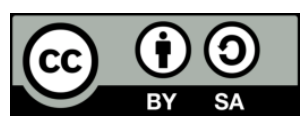

\section{Corresponding Author:}

Nemir Ahmed Al-Azzawi

Department of Mechatronics Engineering

Al-Khwarizmi College of Engineering

University of Baghdad, Iraq

Email: dr.nemir@kecbu.uobaghdad.edu.iq

\section{INTRODUCTION}

Electron microscopy (EM) has appeared as an effective technique to address fundamental knowledge in molecular and cellular biology [1]. Image segmentation algorithms goal is partition pixels into close boundaries corresponding to different essential objects. The major challenge in the visual analysis of microscopy images is the formality of mitochondrial morphologies and nanostructures. The microscopy image segmentation is an important research area that employs different feature detection and representation techniques. Examination and segmentation of electron microscopy images are usually carried out by specialists hand-crafted with knowledge in identifying and clarifying biological structures in the complex grayscale world of electrons. However, efforts to automate the segmentation method were based on supervised machine learning procedures that involve an efficient multiresolution approach and large datasets for training. Improvement has been made in this area, and in progress, but these methods remain limited to defined ideal structures for synapses segmentation of microscopy images [1-4]. 
A shearlet transform is an effective approach to multiscale systems that can capture anisotropic with high efficiency compared to wavelet limitation [5, 6]. The multiscale decomposition shearlet transformation captures the visual information offered by the edges detected in different directions and with multiple scales. The capability of the shearlet transform's inherent directional sensitivity makes it appropriate for characterizing small contours of carcinoma cells. We recall that some recent work using multiscale decomposition for microscopic image analysis has recently appeared in [7-9]. Li et al. [8] proposed the shearlet transform and deep neural networks for the No-reference image quality assessment. Masci et al. [10] has shown the potential for reducing total computational time when training a traditional deep neural network for image segmentation by processing each training image in a single pass rather than patch by patch. Yang et al. [11] used microscopic fecal specimens with digital image processing and an artificial neural network for automatic identification of human helminth eggs. Ho et al. [12] proposed images using three-dimensional convolutional neural networks. David Joon et al. [13] used segmentation of vasculature from fluorescently labelled endothelial cells in multi-photon microscopy images, particularly in the study of tumors. Most of the methods mentioned above used different hand-crafted features to analyze microscopic images. Besides, these hand-crafted features are intended for a defined type of problem, and they are not merely appropriate on other datasets. Furthermore, the scale and the resolution of the acquired microscopic images have to be accounted for to improve accuracy. Fakhry et al. [14] proposed an algorithm for segmenting the EM image by locating dense predictions that combined multidimensional contextual along with full-precision reconstruction. This approach is relying on post-processing techniques that limited the automated visualization of the segmentation. Shen et al. [15] proposed a boundary detection for neuronal by using multistage recursive input in traditional networks. The performance can be enhanced by implementing stage by stage, which limits the memory of the GPU. Chen et al. [16] proposed a deeply supervised contextual neural network for neuronal structure segmentation. Gonda et al. [17] proposed a pixel classifier for the segmentation of EM images by train a deep neural network. This method employs a deep neural network training-feedback loop that captures sparse annotations based on recent and previous annotations. S. Ishii et al. [18] discussed feature reconstruction applications to microscopic images based on multi-frame super-resolution, data-driven image restoration, and deep learning methods that allow training of nonlinear filters for image segmentation. Raju R., T. Maul, et al. [19] suggested using the local contrast slot filling algorithm for a non-learnable, simple, and easy-to-learn algorithm for membrane detection of neuronal slides. Jones C. et al. [20] developed a hierarchical structure for super-pixel incorporation for semi-automatic segmentation in electron microscopy images.

In general, the combined challenges determine the segmentation strategy, and applicable methods have required the development of advanced techniques. In this paper, a robust segmentation of microscopy images based on the shearlet neural network is developed. The proposed method demonstrated that using shearlet coefficients features with the neural network as additional information, the accuracy of segmentation improved compared to the methods that rely on hand-crafted features.

\section{PROPOSED SHEARLET NEURAL NETWORK}

Shearlets were announced in 2006 are a multiscale framework for encoding anisotropic features [5, 6]. The shearlet method is designed from the concept of shearlets through composite dilatation. Moreover, the process that provides a general technique for specific waveforms is in various scales and locations, such as conventional wavelets, and in different directions and with different scaling factors in each coordinate [5]. The shearlets are mainly useful in an amount of image, as shown in several publications (e.g., [8, 21-23]). It is significant for processing applications, such as denoising and feature extraction.

The internal structure of the shearlet neural network is very equivalent to the original neural network. The shearlet neural network consists of the forward-feeding, which contains one or more inputs, one or more hidden layers contains, and the output layer that produces specific outputs for the program, see Figure 1. The hidden layer contains neurons and has specific shearlet activation functions. The structure of a shearlet network shows one output, one input in Figure 2. The hidden layer contains M shearlet activities. The scalar neuron output is a summer of the weighted sum of the shearlet activity outputs.

$$
\begin{aligned}
& y(x)=\sum_{i=1}^{M} w_{i} S H_{a_{i} s_{i} k_{i}}(x)+\bar{y} \\
& S H \psi_{a_{i} s_{i} t_{i}}=\left\langle f, \psi_{a, s, k}\right\rangle, \quad \mathrm{a}>0, \quad s \in \mathbb{R}, \mathrm{t} \in \mathbb{R}^{2}
\end{aligned}
$$


where $\psi_{a, s, k}(x)=\left|\operatorname{det} M_{a s}\right|^{-\frac{1}{2}} \psi\left(M_{a s}^{-1}(x-t)\right)$, and . $M_{a s}=\left[\begin{array}{cc}a & s \\ 0 & \sqrt{a}\end{array}\right]$. The continuous shearlet transform is sampling as $S_{\psi} \quad a_{i} s_{i} t_{i}$. The discrete transform is obtained from an appropriate discrete set [5]. Exactly, $M_{a s}$ is "discretized" as $M_{i j}=B_{i} A^{j}$, where $B=\left(\begin{array}{ll}1 & 1 \\ 0 & 1\end{array}\right)$, shear matrix and $A=\left(\begin{array}{ll}4 & 0 \\ 0 & 2\end{array}\right)$ anisotropic dilation matrix.

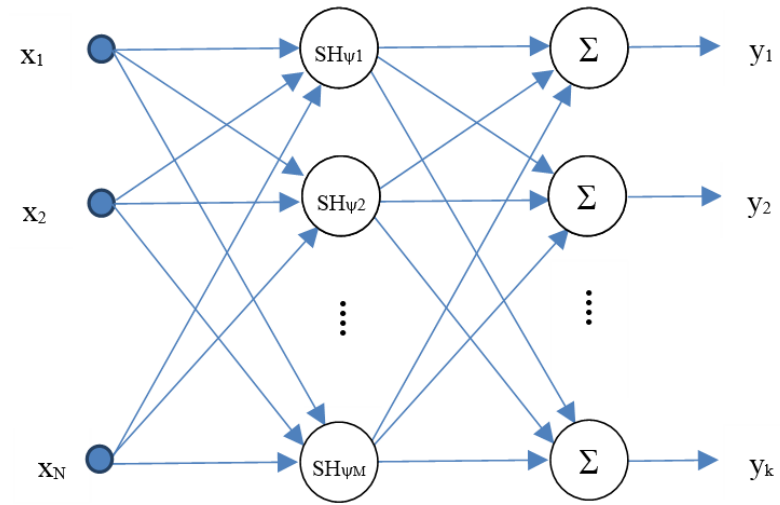

Figure 1. Structure of a shearlet neural network

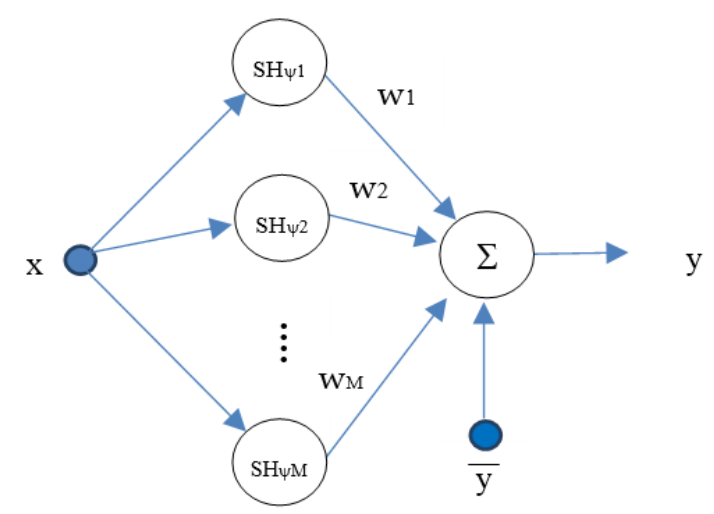

Figure 2. The architecture of the output shearlet network

The general machinery mentioned above constructs a parseval frame of shearlets for $\mathrm{L}^{2}\left(\mathbb{R}^{2}\right)$. Thus, the scaling parameter we use the dyadic sampling $a j=2 j, j \in \mathbb{Z}$. To collect the frequency plane "uniformly", the required a certain number of directions as $\mathrm{j}$ is getting smaller, and, therefore, the set the shear parameter to be $s_{j k}=k \sqrt{a_{j}}=k 2^{\frac{j}{2}}, \mathrm{k} \in \mathbb{Z}$. Lastly, the location parameter is calculated by adjusting the canonical grid $\mathbb{Z}$ to the particular scaling and shear parameter; select $t_{j k m}=S_{s j k} A_{a j m}, m \in \mathbb{Z}^{2}$. Combining all

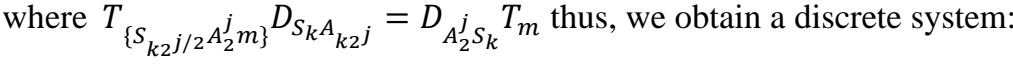

$$
\left\{\psi_{j k m}=D_{A_{2}^{j} S_{k}} T_{m} \psi: j, k \in \mathbb{Z} ; m \in \mathbb{Z}^{2}\right\}
$$

Thus, the functions of the model are the discrete shearlets [5]:

$$
\psi_{a, s, k}(x)=2^{\frac{3 j}{2}} \psi\left(B_{i} A^{j} x-t\right) j \geq 0,-2^{j} \leq l \leq 2^{j} \quad-1, t \in \mathbb{Z}^{2}
$$


The accumulation of the $\bar{y}$ the parameter is to deal with functions that mean is nonzero. The reproducing formula can be approximated to an arbitrary level of detail by sufficiently large $L$ such that:

$$
f(x) \approx \sum_{k}\langle f, \psi\rangle \psi_{a, s, k}(x)
$$

The shearlet neural network output is one or more linear groups of these multidimensional shearlets. The frequency-domain of shearlets $\psi_{a, s, t}$ for variable values of $a$ and $s$ is shown in Figure 3. This shearlet activity is effectively equivalent to a multidimensional shearlet. The hidden layer contains sufficiently entirely of $M$ shearlet activities to cover the domain of the function that is processing and analyzing. The scalar neuron output of the shearlet neural network is expressed in the following manner:

$$
y_{j}=\sum_{i=1}^{M} w_{i j} \psi_{a, s, t}\left(x_{1}, \ldots, x_{N}\right)+\bar{y}_{j} \text { for } j=1, \ldots, K
$$

The parameter $\bar{y}_{j}$ is not needed modification since the mean value of a scaling function is nonzero. The learning algorithm for adjusting the network parameters is function approximation (Zhang and Benveniste proposed an algorithm for this problem application) [24, 25].
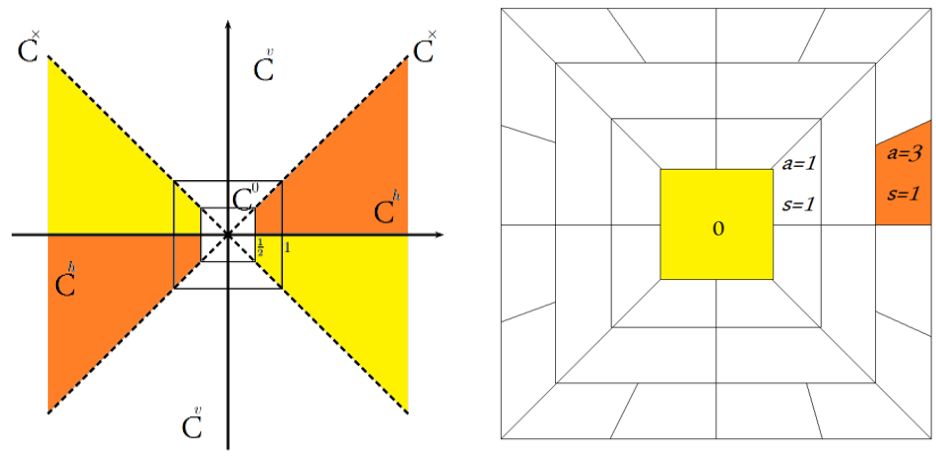

Figure 3. The frequency-domain of shearlets $\psi_{a, s, k}$ for variable values of a and $\mathrm{s}$

Using transform shearlets, which have features including extremely effective rotation and static translation structures, help capture different directional edges in a complex background. The shearlet transform is based on a cone improved discrete shearlet with efficiently maintained shearlets [26]. The frequency domain is separated into two conic sections and a low-frequency portion to solve the drawback of broad shearlet parameters [27]. To obtain good directional selectivity of view shearlets, the horizontal and the vertical cone is defined by [5].

$$
\begin{aligned}
& C^{h}:=\left\{\left(w_{1} ; w_{2}\right) \in \mathbb{R}^{2}:\left|w_{1}\right| \geq \frac{1}{2} ;\left|w_{2}\right|<\left|w_{1}\right|\right\} ; \\
& C^{v}:=\left\{\left(w_{1} ; w_{2}\right) \in \mathbb{R}^{2}:\left|w_{2}\right| \geq \frac{1}{2} ;\left|w_{2}\right|>\left|w_{1}\right|\right\} ; \\
& C^{\times}:=\left\{\left(w_{1} ; w_{2}\right) \in \mathbb{R}^{2}:\left|w_{1}\right| \geq \frac{1}{2} ;\left|w_{2}\right| \geq \frac{1}{2} ;\left|w_{2}\right|=\left|w_{1}\right|\right\} ; \\
& C^{0}:=\left\{\left(w_{1} ; w_{2}\right) \in \mathbb{R}^{2}:\left|w_{1}\right|<1 ;\left|w_{2}\right|<1\right\} ;
\end{aligned}
$$

It would be appropriate to simplify the notation to combine vertical and horizontal transforms $(d=0$, 1) by renaming the orientation index $s$ as in (8).

$$
\operatorname{SH} \psi f(a, s, k)=\left\{\begin{array}{cc}
\operatorname{SH}^{(0)} f\left[a, s-1-2^{\mathrm{a}}, k\right], & 1 \leq s \leq 2^{a+1} \\
\mathrm{SH}^{(1)} f\left[a, 3\left(2^{a}\right)-s, k\right], & 2^{a+1}<s \leq 2^{a+2}
\end{array}\right\}
$$

where $a>0$ is the scale, $s$ is the orientation, and $k$ is the location. The directional scale-space decomposition of image $f$ is providing by the shearlet decomposition. Applying shearlet transform to the image $f \in L^{2}\left(\mathbb{Z}^{2}\right)$ is given by:

Microscopy images segmentation algorithm based on shearlet neural network (Nemir Ahmed Al-Azzawi) 
$f \rightarrow \operatorname{SH} \psi f(a, s, k)$,

The whole number of shearlet decomposition created in shearlet transform is $\sum_{a=0}^{t n a-1} 2^{\left[\frac{\mathrm{a}}{2}\right]+2}$, where tna denotes the scales of the shearlet transform. The image boundary features $b_{j} f$ at the level $a$ are then given by:

$$
b_{\mathrm{a}}^{2} \mathrm{f}[\mathrm{k}]=\left(\sum_{\mathrm{a}} \mathrm{SH}^{1} \mathrm{f}[\mathrm{a}, \mathrm{s}, \mathrm{k}]\right)^{2}+\left(\sum_{\mathrm{a}} \mathrm{SH}^{2} \mathrm{f}[\mathrm{a}, \mathrm{s}, \mathrm{k}]\right)^{2}
$$

The third layer's output is the weighted sum of the image boundary features that segment between the membrane and cells. Shearlet network training involves minimizing the traditional least-squares to estimate the cost function:

$$
E=\sum_{i=1}^{n}\left(y_{i}-o_{i}\right)^{2}
$$

where $o_{i}$ is the optimal output of the $i$ th input vector, and $n$ is the number of training samples for each group. Figure 4 is the SNN training algorithm's epoch, which illustrations the error is nearly 0,04 by the 12 iterations.

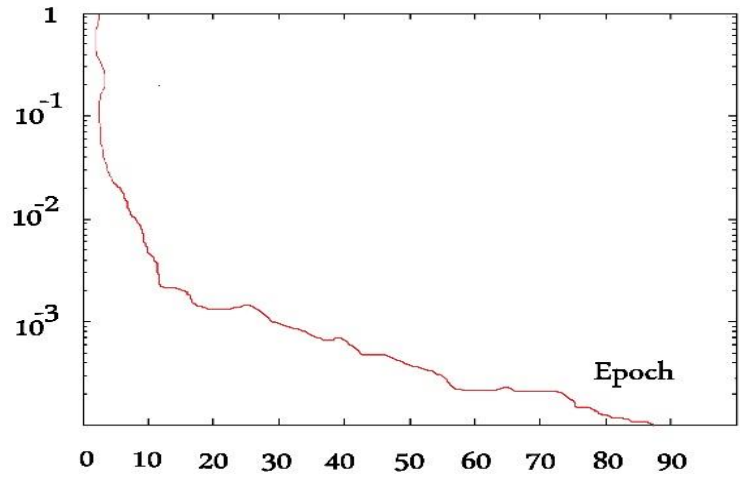

Figure 4. The epochs of the SNN training algorithm

\section{RESULTS AND DISCUSSION}

The shearlet neural network is implemented using MATLAB on a PC consists of the Intel Core i78550U processor type with a 16 GB main memory and an NVIDIA 4GB GDDR5 GPU. The proposal is validated using the publicly available dataset provided by ISBI 2012 organizers of the ISBI 2012 electron microscopy image [28] with ground-truth labelled by a human expert. The data set consists of two groups of $512 \times 512 \times 30$, one for training and one for testing. The image dataset represents two parts of the ventral nerve cord of the Drosophila larva. Electronic microscopic images usually share similar materials independent of the direction in microstructures such as membrane, synapse, intracellular, mitochondria, glia, extracellular, and axons. Implementing a training stack of basic fact segmentation is provided by annotation manually. Practical resolution measures are the average percentage of pixels that are properly segmented for both foreground and background units [29].

The proposed segmentation method's overall efficiency performance was estimated by F-score, recall, precision, and accuracy. The combined quantitative analysis is performed based on the pixel similarity of the resulting segmented image versus the manually segmented image. The precision is represented repeatability of segmentation, taking into account all subjective actions required to produce the product [30-33]. The possible results are expressed as familiar images correctly classified (FICC), familiar images abnormal classified as (FIAC), strange images correctly classified (SICC), and strange images abnormal classified (SIAC).

$$
\text { Accuracy }=\frac{\sum \text { SICC }+\sum \text { FICC }}{\sum \text { SICC }+\sum \text { FICC }+\sum \text { FIAC }+\sum \text { SIAC }} \times 100
$$


Precision $=\frac{\sum \text { FICC }}{\sum \text { FICC }+\sum \text { SICC }} \times 100$

Recall $=\frac{\sum \text { FICC }}{\sum \text { FICC }+\sum \text { SIAC }} \times 100$

$\mathrm{F}-$ score $=($ Recall $*$ Precision $* 2) /($ Recall + Precision $)$

Adapted Rand error $=1-(\mathrm{F}-$ score $)$

Table 1 shows the segmentation ideality-based accuracy, precision and recall, and F-score. F-score is an indication of perfect or unperfect segmentation, usually 1 for ideal segmentation. The adapted Rand error is among 0 and 1, generally 0 for ideal segmentation. The Segmentation accuracies obtained in the proposed algorithm are compared by the existing technique obtained by Raju et al. [19] (the local contrast slot filling and classical-based), Jones et al. [20] (semi-automatic segmentation and deep learning-based), all used the same dataset ISBI 2012 electron microscopy image [28]. We can see from Table 1, the performance was good in segmentation due to the dataset having an even distribution for large structures. The proposed method used the 30 slices in the dataset to train and test with their corresponding ground truth. F-score covers the general information of the segmentation image. Thus, provide a better measurement of segmentation performance, whereas accuracy, recall, and precision are straight depending on pixels similarity comparison. It can be noticed through Table 1, that for a total average of 30 images, the proposed method is proven to be the acceptable in segmenting the entire area in microscopic images and related regions with segmentation accuracy of $96.2 \%$, F-score 0.962 and Rand error 0.038 . By comparing the results, the proposed method is found optimum supported for segmentation performance. Figure 5, shows the proposed SNN segmentation result.

Table 1 . The quality of the segmented image is represented by values of accuracy, precision, and recall, and

F-score for (average for 30 images)

\begin{tabular}{ccccc}
\hline Methods & Accuracy & Precision & Recall & F-score \\
\hline Proposed & $96.2 \%$ & $95.11 \%$ & $97.33 \%$ & 0.9620 \\
(Classical-based) [19] & & $64.29 \%$ & $79.74 \%$ & 0.7107 \\
(Deep learning-based) [20] & $86.9 \%$ & $90.80 \%$ & $83.40 \%$ & 0.8694 \\
\hline
\end{tabular}

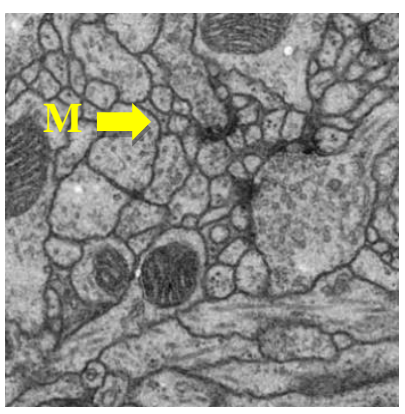

(a)

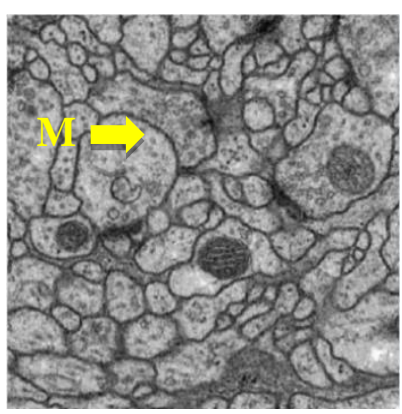

(b)

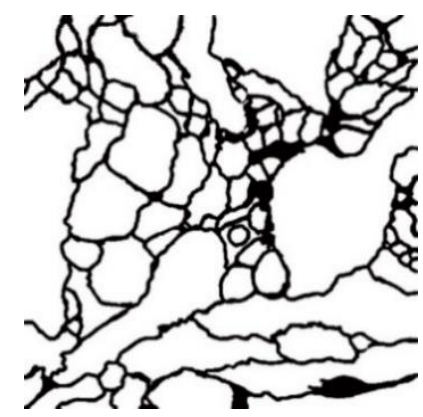

(c)

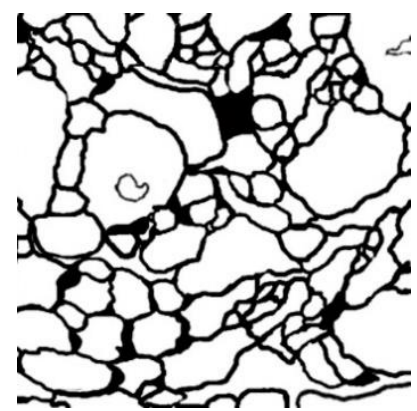

(d)

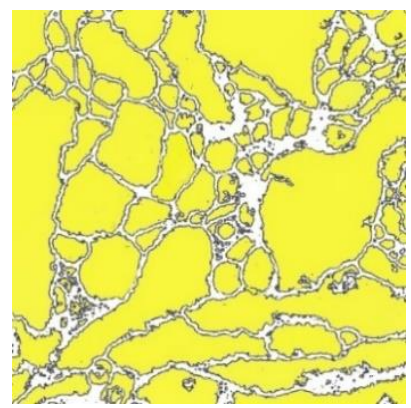

(e)

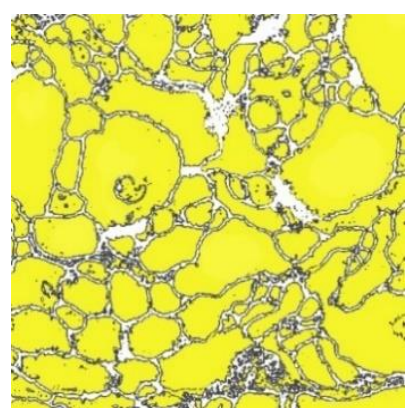

(f)

Figure 5. Image data sets used for SNN training and testing with their corresponding ground truth labels M: membrane+synapse, (a) (b) Microscopic test image; (c) (d) Ground-truth, (e) (f) Specify segmentation result 
The recall and precision provide further information about the type of error involved in segmented images. For example, when the recall is low and the precision is high, this indicates that the image was over segmented. Conversely, it demonstrates that the image is under segmented, and then it should be separate. Therefore, we notice in the results method Jones et al. [20] that the image was under-segmented. Figure 5, It is evident that the proposed SNN standard segmentation acceptably detects membranes. The segmentation result based on the SNN is accurate compared to ground-truth. Our approach outperforms all these research methods; thus, the paper's proposed is applicable in the microscopy image segmentation.

\section{CONCLUSION}

In this paper, we presented a new shearlet neural network structure for image segmentation, specifically targeting microscopic images. A mathematical technique for segmentation an electron microscopic image by calculating the dense predictions gathered across multiple scales with complete and accurate reconstructions. Our approach has performed promisingly with the best precision. Automating this process can help pathologists to have a faster and more reliable diagnosis. The proposed method's main advantages are that it does not make any assumptions about the visual features. The shearlet transform is considered as a specific mathematical tool for extracting features. We believe that the proposed approach could work well for microscopic image segmentation tasks. The experimental results on the 2D microscopy image segmentation confirm the benefits of the proposed method.

\section{REFERENCES}

[1] J.-J. Fernandez, "Computational methods for electron tomography," Micron, vol. 43, no. 10, pp. 1010-1030, 2012.

[2] O. Ronneberger, P. Fischer, and T. Brox, "U-net: Convolutional networks for biomedical image segmentation," Springer, Cham, pp. 234-241, 2015.

[3] M. B. Ahrens, M. B. Orger, D. N. Robson, J. M. Li, and P. J. Keller, "Whole-brain functional imaging at cellular resolution using light-sheet microscopy," Nature methods, vol. 10, pp. 413-420, 2013.

[4] E. Jurrus, A. R. C. Paiva, S. Watanabe, J. R. Anderson, B. W. Jones, R. T. Whitaker, E. M. Jorgensen, R. E. Marc, and T. Tasdizen, "Detection of neuron membranes in electron microscopy images using a serial neural network architecture," Medical Image Analysis, vol. 14, no. 6, pp. 770-783, 2010.

[5] K. Guo, G. Kutyniok, and D. Labate, "Sparse multidimensional representations using anisotropic dilation and shear operators," ed: Wavelets and Splines (Athens, GA, 2005), Nashboro Press, Nashville, TN, 2006.

[6] K. Guo and D. Labate, "The construction of smooth Parseval frames of shearlets," Mathematical Modelling of Natural Phenomena, vol. 8, no. 1, pp. 82-105, 2013.

[7] Y. Guo, A. S. Ashour, and B. Sun, "A novel glomerular basement membrane segmentation using neutrsophic set and shearlet transform on microscopic images," Health information science and systems, vol. 5, no. 1, p. 5, 2017.

[8] Y. Li, L.-M. Po, X. Xu, L. Feng, F. Yuan, C.-H. Cheung, and K.-W. Cheung, "No-reference image quality assessment with shearlet transform and deep neural networks," Neurocomputing, vol. 154, pp. 94-109, 2015.

[9] J. Antonello, A. Barbotin, E. Z. Chong, J. Rittscher, and M. J. Booth, "Multiscale sensorless adaptive optics: application to stimulated emission depletion microscopy," Optics Express, vol. 28, no. 11, pp. 16749-16763, 2020.

[10] J. Masci, A. Giusti, D. Ciresan, G. Fricout, and J. Schmidhuber, "A fast learning algorithm for image segmentation with max-pooling convolutional networks," 2013 IEEE International Conference on Image Processing, Melbourne, VIC, pp. 2713-2717, 2013.

[11] Yoon Seok Yang, Duck Kun Park, Hee Chan Kim, Min-Ho Choi, and Jong-Yil Chai, "Automatic identification of human helminth eggs on microscopic fecal specimens using digital image processing and an artificial neural network," in IEEE Transactions on Biomedical Engineering, vol. 48, no. 6, pp. 718-730, June 2001.

[12] D. J. Ho, C. Fu, P. Salama, K. W. Dunn, and E. J. Delp, "Nuclei Segmentation of Fluorescence Microscopy Images Using Three Dimensional Convolutional Neural Networks," in 2017 IEEE Conference on Computer Vision and Pattern Recognition Workshops (CVPRW), pp. 834-842, 2017.

[13] R. Bates, B. Irving, B. Markelc, J. Kaeppler, G. Brown, R. J. Muschel, M. Brady, V. Grau, and J. A. Schnabel, "Segmentation of vasculature from fluorescently labeled endothelial cells in multi-photon microscopy images," in IEEE Transactions on Medical Imaging, vol. 38, no. 1, pp. 1-10, Jan. 2019.

[14] A. Fakhry, T. Zeng, and S. Ji, "Residual deconvolutional networks for brain electron microscopy image segmentation," in IEEE Transactions on Medical Imaging, vol. 36, no. 2, pp. 447-456, Feb 2017.

[15] W. Shen, B. Wang, Y. Jiang, Y. Wang, and A. L. Yuille, "Multi-stage multi-recursive-input fully convolutional networks for neuronal boundary detection," Center for Brains, Minds and Machines (CBMM), 2017.

[16] H. Chen, X. Qi, J.-Z. Cheng, and P.-A. Heng, "Deep contextual networks for neuronal structure segmentation," in AAAI'16: Proceedings of the Thirtieth AAAI Conference on Artificial Intelligence, pp. 1167-1173, 2016.

[17] F. Gonda, V. Kaynig, T. R. JOnes, D. Haehn, J. W. Lichtman, T. Parag, and H. Pfister, "ICON: An interactive approach to train deep neural networks for segmentation of neuronal structures," 2017 IEEE 14th International Symposium on Biomedical Imaging (ISBI 2017), Melbourne, VIC, pp. 327-331, 2017.

\[18] S. Ishii, S. Lee, H. Urakubo, H. Kume, and H. Kasai, "Generative and discriminative model-based approaches to microscopic image restoration and segmentation," Microscopy, vol. 69, no. 2, pp. 79-91, 2020. 
[19] R. Raju, T. Maul, and A. Bargiela, "Local contrast hole filling algorithm for neura slices membrane detectionLCHF," 2014 IEEE Symposium on Computer Applications and Industrial Electronics (ISCAIE), Penang, pp. 146151. 2014

[20] C. Jones, T. Liu, N. W. Cohan, M. Ellisman, and T. Tasdizen, "Efficient semi-automatic 3D segmentation for neuron tracing in electron microscopy images," Journal of neuroscience methods, vol. 246, pp. 13-21, 2015.

[21] H. Rezaeilouyeh, M. H. Mahoor, S. M. Mavadati, and J. J. Zhang, "A microscopic image classification method using shearlet transform," 2013 IEEE International Conference on Healthcare Informatics, Philadelphia, PA, pp. 382-386, 2013.

[22] K. Guo and D. Labate, "Optimally sparse multidimensional representation using shearlets," SIAM Society for Industrial and Applied Mathematics, vol. 39, no. 1, pp. 298-318, 2007.

[23] C. Deng, J. Liu, W. Tian, S. Wang, H. Zhu, and S. Zhang, "Image super-resolution reconstruction based on L1/2 sparsity," Bulletin of Electrical Engineering and Informatics, vol. 3, no. 3, pp. 155-160, 2014.

[24] Qinghua Zhang, "Using wavelet network in nonparametric estimation," in IEEE Transactions on Neural Networks, vol. 8, no. 2, pp. 227-236, March 1997.

[25] Q. Zhang and A. Benveniste, "Wavelet networks," in IEEE Transactions on Neural Networks, vol. 3, no. 6, pp. 889-898, Nov. 1992.

[26] G. Kutyniok, W.-Q. Lim, and X. Zhuang, "Digital shearlet transforms," in Shearlets, ed: Springer, pp. 239-282, 2012.

[27] S. Häuser and G. Steidl, "Fast finite shearlet transform," arXiv preprint arXiv:1202.1773, pp. 1-38, 2012.

[28] S. H. Arganda-Carreras I, Cardona A, and Schindelin J, "Segmentation of neuronal structures in EM stacks challenge-ISBI 2012," 2012. [Online]. Available at: http://brainiac2.mit.edu/isbi_challenge/home.

[29] O. Lezoray and H. Cardot, "Cooperation of color pixel classification schemes and color watershed: a study for microscopic images," in IEEE Transactions on Image Processing, vol. 11, no. 7, pp. 783-789, July 2002.

[30] C. E. Metz, "Basic principles of ROC analysis," in Seminars in Nuclear Medicine, pp. 283-298, 1978.

[31] M.-J. Zhao, N. Edakunni, A. Pocock, and G. Brown, "Beyond Fano's inequality: bounds on the optimal F-score, BER, and cost-sensitive risk and their implications," Journal of Machine Learning Research, vol. 14, pp. 10331090, 2013.

[32] N. A. Al-Azzawi and M. K. Sabir, "An superior achievement of brain tumor detection using segmentation based on F-transform," 2015 World Symposium on Computer Networks and Information Security (WSCNIS), Hammamet, pp. $1-5,2015$.

[33] N. A. Al-Azzawi, "Color medical imaging fusion based on principle component analysis and F-transform," Pattern Recognition and Image Analysis, vol. 28, pp. 393-399, 2018.

\section{BIOGRAPHY OF AUTHOR}

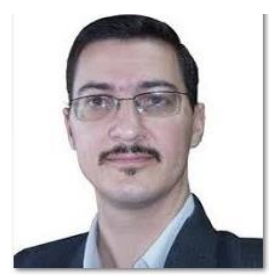

Nemir Ahmed Al-Azzawi Associate Professor at the Department of Mechatronics, Al Khwarizmi College of Engineering, University of Baghdad. He received a Bachelor in Electrical Engineering (Hons), Master of Science Electronics and Communication from the University of Baghdad, and a Ph.D. degree from the School of Electrical and Electronics, Universiti Sains Malaysia (USM). He has been the Head of the Department since Feb 2018 and is a member of the College Senate. His research interests include Medical Image Processing, Computer Vision, Visual Cryptography, OFDM, Image registration and fusion, genetic algorithms in communications. 